\title{
Evaluation of systemic inflammatory responses in cholecystectomy by means of access. Single-port umbilical incision, transvaginal NOTES, laparoscopy and laparotomy ${ }^{1}$
}

\author{
Elesiário Marques Caetano Júnior ${ }^{\mathrm{I}}$, Josiel Paiva Vieira ${ }^{\mathrm{II}}$, Rita Maria A Monteiro Moura-Franco ${ }^{\mathrm{III}}$, Rogerio Aoki Fuziy ${ }^{\mathrm{IV}}$,

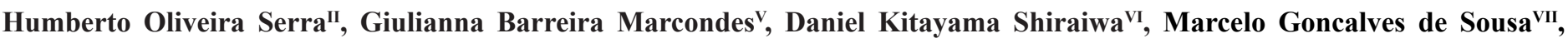 \\ Manoel João Batista Castello Girão ${ }^{\mathrm{VIII}}$, Gaspar de Jesus Lopes-Filho ${ }^{\mathrm{IX}}$, Marcelo Moura Linhares ${ }^{\mathrm{X}}$
}

DOI: http://dx.doi.org/10.1590/S0102-865020150100000007

IFellow Master degree, Postgraduate Program in Interdisciplinary Surgical Sciences, Division of Surgical Gastroenterology, Department of Surgery, Universidade Federal de São Paulo (UNIFESP), Brazil. Conception and design of the study; acquisition, analysis and interpretation of data; drafting the article; final approval of the version to be published.

IIPhD, Assistant Professor, Division of Surgical Gastroenterology, Department of Surgery, Universidade Federal do Maranhão (UFMA), Sao Luis-MA, Brazil. Acquisition of data, final approval of the version to be published.

IIIPhD, Assistant Professor, Division of Surgical Gastroenterology, Department of Surgery, UNIFESP, Sao Paulo-SP, Brazil. Acquisition of data, final approval of the version to be published.

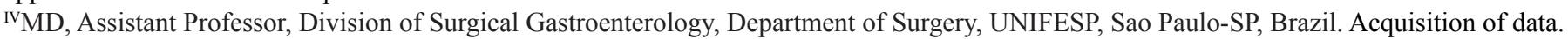

${ }^{v}$ Graduate student, School of Medicine, UNIFESP, Sao Paulo-SP, Brazil. Grant from Institutional Program for Scientific Initiation (PIBIC) of the National Council of Scientific and Technological Development (CNPq), Ministry of Science, Technology and Inovation, Brazil. Conception and design of the study, acquisition of data.

${ }^{\mathrm{VI}}$ Graduate student, School of Medicine, UNIFESP, Sao Paulo-SP, Brazil. Grant from Institutional Program for Scientific Initiation (PIBIC) of the National Council of Scientific and Technological Development (CNPq), Ministry of Science, Technology and Inovation, Brazil. Conception and design of the study.

VIIPhD, Assistant Professor, Division of Surgical Gastroenterology, Department of Surgery, Universidade Federal da Paraiba (UFPB), Joao Pessoa-PB, Brazil. Acquisition of data, final approval of the version to be published.

VIIIPhD, Chairman, Division of Gynecology, Department of Surgery, UNIFESP, Sao Paulo-SP, Brazil. Research Productivity Level 2, Grant from National Council of Scientific and Technological Development (CNPq), Ministry of Science, Technology and Inovation, Brazil. Intellectual content of the study, final approval of the version to be published.

IXPhD, Chairman, Division of Surgical Gastroenterology, Department of Surgery, UNIFESP, São Paulo-SP, Brazil. Research Productivity Level 2, Grant from National Council of Scientific and Technological Development (CNPq), Ministry of Science, Technology and Inovation, Brazil. Intellectual content of the study, final approval of the version to be published.

xPhD, Associate Professor, Division of Surgical Gastroenterology, Department of Surgery, UNIFESP, Sao Paulo-SP, Brazil. Conception and design of the study; acquisition, analysis and interpretation of data; drafting the article; final approval of the version to be published.

\begin{abstract}
PURPOSE: To evaluate and compare clinical and inflammatory responses to the surgical trauma caused by cholecystectomy via several access approaches: single-port umbilical incision (SILS), transvaginal natural orifice transluminal endoscopic surgery (NOTES), laparoscopy, and Laparotomy.

METHODS: Twenty-eight female pigs were equally divided into four groups and submitted to cholecystectomy by single-port umbilical incision, transvaginal NOTES, laparoscopy, or Laparotomy. An additional five animals served as controls (sham group). Animals were monitored perioperatively regarding anesthesia and surgical procedure times, as well as for the presence of complications. Postoperatively, they were evaluated regarding time to ambulation and feeding, and the presence of clinical events. Procalcitonin, C-reactive protein (CRP), and AQUI feron-gamma (IFN- $\gamma$ ) measurements were performed before surgery and immediately, two days, and seven days after surgery. Animals were sacrificed and necropsied at seven days after surgery.

RESULTS: All procedures were successfully performed as proposed in each group. Only minor complications, such as gallbladder perforation and bleeding from the liver bed, were observed during surgery in all groups. The vaginal NOTES group showed higher anesthesia and surgical procedure times compared to the other groups $(\mathrm{p}<0.001)$. No other between-group differences in perioperative or postoperative times, clinical evolution, or serum inflammatory markers were observed. Only adhesions were found on necropsy, with no differences between groups.
\end{abstract}

CONCLUSION: The single-port umbilical and transvaginal NOTES access approaches were feasible and safe compared to laparoscopic and laparotomy for cholecystectomy.

Key words: Cholecystectomy, Laparoscopic. Laparotomy. Cytokines. Swine. 


\section{Introduction}

Surgical trauma induces immediate neuroendocrine, metabolic, and immunologic responses in the body, aimed at controlling tissue damage, combatting infection, and promoting processes to repair and restore damaged tissues through inflammatory and anti-inflammatory factors ${ }^{1}$. The intensity of this response is directly proportional to the magnitude of the surgical trauma, but an excessive response to trauma can be harmful to the patient ${ }^{2}$. Elevated levels of proinflammatory cytokines, such as tumor necrosis factor-alpha (TNF- $\alpha$ ) and interleukin-1 beta (IL$1 \beta$ ), are associated with increased occurrences of bacterial growth, systemic inflammatory response syndrome, multiple organ and systems failure, and respiratory distress syndrome ${ }^{3}$. A feedback mechanism tends to prolong this state of hyperinflammation, although it can, paradoxically, cause immunosuppression ${ }^{4}$. This hyperinflammation-immunosuppression cycle is also proportional to the level of initial aggression ${ }^{4}$. Clinically, this cycle can increase postoperative morbidity and mortality by sepsis, organ failure, and death $^{5}$.

Over the past 30 years, a major challenge in every surgical field has been how to achieve a balance between minimizing surgical trauma and ensuring operative feasibility. Minimally invasive surgery (MIS) was one of the most important surgical developments in the 20th century ${ }^{6}$. Laparoscopic surgery broke paradigms and revolutionized the world of surgery, becoming the gold standard for the surgical treatment of digestive system diseases $^{6,7}$. Comparisons of open abdominal surgery procedures and MIS techniques have revealed that laparoscopic surgeries are generally associated with less trauma, less pain, shorter hospital stays, better postoperative recovery, and better aesthetic results for patients ${ }^{8}$. For these reasons, there has been growing interest in this procedure, as evidenced by the increasing publication of articles related to minimally invasive laparoscopy ${ }^{9}$.

Natural Orifice Translumenal Endoscopy Surgery (NOTES), also known as "scarless" or "third-generation" surgery, is a new surgical concept that seeks to reduce invasion of the abdominal cavity by accessing it through one of the body's natural orifices (e.g., vagina, bladder, colon, or stomach) using only a flexible endoscope ${ }^{10,11}$. Theoretical advantages of NOTES derive from its complete elimination of the need for an abdominal incision and its minimal invasiveness compared to other approaches ${ }^{11}$. NOTES avoids problems related to the operative wound, such as pain, infection, herniation, and adherence ${ }^{12}$, thereby leading to a faster postoperative recovery, shorter hospital stay, and better aesthetic result ${ }^{13}$. NOTES can also have a favorable immunological impact on the patient ${ }^{14}$.
Experimental studies have evaluated the inflammatory response provoked by NOTES compared with laparoscopic access, based on measurements of acute-phase systemic cytokines and proteins. The authors studied the physiologic impact of NOTES by measuring levels of the proinflammatory cytokines IL-1 $\beta$, IL- 6 , and TNF- $\alpha$ in 37 pigs treated by sham surgery or peritonioscopy with NOTES, laparoscopy or Laparotomy. Pigs treated by NOTES peritonioscopy showed similar TNF- $\alpha$ levels compared to pigs treated by laparoscopy or Laparotomy. Although the authors were unable to obtain consistent measurements for IL- $1 \beta$ and IL- 6 for any of the surgical groups, the TNF- $\alpha$ levels were lower in the postoperative period, indicating that NOTES had an immunomodulation effect that was not found with the other access approaches. Authors have also examined inflammatory markers with different approaches for cholecystectomy. Studies comparing open with laparoscopic cholecystectomy demonstrated a significant increase in IL-6 and C-reactive protein (CRP) for Laparotomy $^{4,15}$.

The success of NOTES has encouraged the development of new laparoscopic technologies, including multiple-port articulated instruments, and has indirectly contributed to the development of single-port surgery, as an alternative to surgery through natural orifices ${ }^{16}$. In 1997, Giuseppe Navarra of Italy published the first report on single-port cholecystectomy ${ }^{17}$. In a non-random study, the authors compared open and single-port cholecystectomy in terms of the impact of surgical trauma on the levels of inflammatory markers. They did not find any statistically significant differences in IL-6 and CRP levels between the two approaches $^{15}$.

There is a need to study the clinical response and the magnitude of trauma provoked by each of these approaches. In this study, cholecystectomy performed by transvaginal NOTES access or a single-port umbilical incision was compared to traditional procedures, such as conventional laparoscopy and open surgery (laparotomy). The clinical and inflammatory responses to surgical trauma were compared among the groups.

\section{Methods}

The protocol for this study was approved by the Research Ethics Committee at the Universidade Federal de São Paulo (CEP 0755/07) and Hospital Israelita Albert Einstein. The objective was to study the clinical and inflammatory response to surgical trauma in an experimental animal model subjected to cholecystectomy via single-port umbilical incision, transvaginal NOTES endoscopy, laparoscopic, and laparotomic approaches. Serum measurements 
of proinflammatory cytokines, including procalcitonin (PCT), interferon gamma (IFN- $\gamma$ ), and CRP, were compared among the groups. Thirty-three 33 Large White pigs (Sus scrofusdomesticus), three months of age and weighing 20 to $35 \mathrm{~kg}$, were randomly divided into five groups: single-port (seven animals); transvaginal NOTES (seven animals); laparoscopy (seven animals); laparotomy (seven animals); and sham (five animals).

\section{Animal preparation and operative technique}

The preparation technique for animals was based on the consensus for care during animal experimentation from the International Association for the Assessment and Accreditation of Laboratory Animal Care (AAALAC), as described previously ${ }^{18}$.

Transvaginal NOTES access. Animals in this group were initially placed in the Trendelenburg position. Peri- and intravaginal antisepsis were performed with topical chlorhexidine. A plastic sheet was fixed lengthwise, separating the perianal region from the vagina, and sterile operating fields were placed. The back wall of the vagina was identified, and an incision was made in the epithelium and muscle with a diathermic probe. As soon as the probe penetrated the muscle bed, the electrocauterizer was turned off, and the probe was forced into the peritoneal cavity. A pneumoperitoneum was created, with the pressure being continuously monitored via a tube attached from one of the endoscope's working channels to the pressure monitor. The pneumoperitoneum was maintained with ambient air, generated by an endoscopic inflation bulb. Pressure and deflation were monitored intermittently using surgical equipment when convenient and/or at the anesthesiologist's request.

A guide-wire was introduced into the peritoneal cavity, to guide the passage of the dual-channel endoscope. The endoscope and two grasp-type arciform forceps, $70 \mathrm{~cm}$ in length and $5 \mathrm{~mm}$ in diameter, were guided under direct vision to an area near the right hypochondrium. The original position of the animal was inverted (proclive), and the abdominal cavity was inventoried. The bile duct was located, clamped, and pulled back with extra-long rigid forceps, positioned in the vagina externally to the endoscope. One forceps was used to grab and raise the base of the bile duct. Another was used to grab, traction, and triangulate the abdominal end of the uterus. The duct and cystic artery were dissected with a hook-type diathermic endoscopic forceps, which was introduced through the working channel for the endoscope. Up-down and leftright movements were used at the end of the device to enable the dissection movements of the endoscopic forceps.

After the artery and the cystic duct were completely dissected, these structures were proximally and distally occluded with endoscopic clips and sectioned with endoscopic scissors. The bile duct was dissected from the liver bed with the endoscopic cauterizer, using the hook-type diathermic endoscopic forceps positioned in the working channel for the endoscope. Finally, hemostasis of the vascular bed was performed, the cavity was reviewed, and the surgical piece was withdrawn through the vaginal opening, together with the endoscope and forceps. The vaginal cupola was left open.

Laparoscopic access. Asepsis and abdominal antisepsis were performed with topical chlorhexidine. A pneumoperitoneum was made via the periumbilical incision, through which a $10-\mathrm{mm}$ trocar was introduced. The abdominal cavity was inventoried. Three other access points were introduced under laparoscopic view: epigastric $(10 \mathrm{~mm})$, right flank $(5 \mathrm{~mm})$, and right hypochondrium $(5 \mathrm{~mm})$. The classic retrograde process was used to perform the technique. Dissection began by isolating and clipping the artery and the cystic duct, which were tied together with titanium clips. The bile duct was dissected from the liver bed and removed. Finally, the aponeuroses of the trocar's openings were closed with zero vicryl, and the skin was closed with a continuous point of 3-0 mononylon. The intra-abdominal pressure (IAP) was continuously maintained at $14 \mathrm{mmHg}$.

Laparotomy. Abdominal trichotomy was performed with an electric appliance. Abdominal antisepsis was ensured with topical chlorhexidine, and the operating fields were placed. A right subcostal incision of 8 to $10 \mathrm{~cm}$ was made. The wall was opened using the electrocauterizer to section the oblique external, internal, and cross muscles of the abdomen, as well as the peritoneum. The abdominal cavity was inventoried. The anterograde technique was used to perform the cholecystectomy, which consisted of dissection and repair of the artery and the cystic duct, dissection of the bile duct, hemostasis of the liver bed, and, finally, ligature of the cystic duct and removal of the bile duct. The abdominal cavity and hemostasis of the liver bed were carefully reviewed, and the abdominal cavity was closed by planes, using zero vicryl and 3-0 mononylon threads.

Single-port access. Asepsis and abdominal antisepsis were performed with topical chlorhexidine, and the sterile surgical fields were placed. A 2.5-cm-long periumbilical incision was made, through which the Edlo ${ }^{\circledR} 10-\mathrm{mm}$ single trocar access device was introduced. This device has three $5-\mathrm{mm}$ and one $10-\mathrm{mm}$ entrance and an inflatable-balloon fixing system. It measures $35 \mathrm{~mm}$ wide, and was molded to the incision.

The following instruments were used to perform the procedure: articulated forceps developed for this access; a 
biarticulated dissector; hook-shaped forceps with articulation at the distal end; and arc-shaped scissors, especially made to be used in a single port. The possibility of using an electric current was available. The pneumoperitoneum pressure was maintained at $14 \mathrm{mmHg}$ during surgery, by using a laparoscopic insufflator connected to a specific access approach for this purpose. The surgical table remained in the reverse Trendelenburg position. The surgical team consisting of a surgeon and an assistant who stood at the animal's feet. The surgical rack stood at the head of the surgical table.

Three forceps were introduced through the 5-mm opening of the single-access device: one articulated forceps was used to apply traction of the base of the vesicle, and two forceps were used to dissect the vesicle from the liver bed. The retrograde technique was used, with the vesicle dislocated in a way similar to that used in the conventional laparoscopic technique. The cystic duct and cystic artery were dissected and tied off with titanium clips and a 5-mm clipper. The bile duct was removed from the liver bed by using hook-shaped forceps with an electrocauterizer. The vesicle was removed from the abdominal cavity through the trocar hole. Finally, the single-port umbilical incision was sutured with an approximation of aponeurosis, using zero vicryl thread, and the nearby skin with a continuous point of 3-0 mononylon.

Sham procedure. The sham group consisted of five animals that underwent the same preoperative and anesthetic procedures, including medication, monitoring, and blood sample collection, as the surgical groups. The sham group remained anesthetized for the average anesthetic time of the other study groups but did not have any surgical intervention, neither to perform a cholecystectomy nor to obtain the respective access approaches.

\section{Perioperative and postoperative evaluations}

After the planned anesthetic times, the anesthesiologists reanimated the animals and returned them to their bays. During the surgery, the following parameters were monitored: anesthetic, operative, and extubation times; cardiopulmonary monitoring parameters, such as heart rate (HR), respiratory rate (RR), and $\mathrm{O}_{2}$ arterial saturation $\left(\mathrm{O}_{2} \mathrm{SA}\right)$; occurrence of perioperative complications; and anesthetic recovery time. The animals were kept alive for seven days postoperation (DPO). Immediately after the surgery, the deambulation and refeeding times were determined. On a daily basis, the following aspects were analyzed: animal behavior, food and water intake, and presence of clinical complications. The goal of these analyses was to determine the presence of infection, dehiscence of the operative wound, evisceration, digestive fistula, and mortality.

\section{Sample collection and preparation}

In the immediate postoperative period, shortly after etomidate was injected, around $15 \mathrm{~mL}$ of blood were collected by deep puncture of the iliac vein under ultrasonographic repair. Additional blood samples were collected at the end of the anesthetic procedure and on 2 and 7 DPO. The final two samples were collected with the animal sedated using a solution of $1 \%$ acepromazine $(1 \mathrm{mg} / \mathrm{kg})$ and midazolan $(0.2 \mathrm{mg} / \mathrm{kg})$, intramuscularly.

Immediately after collection, the blood was divided into two flasks for storage and centrifuged at $4000 \mathrm{rpm}$. The plasma was collected, distributed by pipettes into two Eppendorf tubes, and frozen at $-80^{\circ} \mathrm{C}$. Samples were sent to a specialized laboratory for determination of the PCT, CPR, and IFN- $\gamma$ levels by enzyme-linked immunosorbent assay (ELISA) with the Porcine Procalcitonin (lot: B25120363), Pig C-Reactive Protein (Life Diagnostics, Inc., Cat. No. 2210-6), and Porcine IFN- $\gamma$ (Cat. No. CSB-E06794p) ELISA test kits, respectively.

\section{Macroscopic evaluation by necropsy}

At 7 DPO, the animals were euthanized and laparotomized. The abdominal cavity was inventoried, and the presence, location, and characteristics of peritonitis, abscess, fistula, bleeding, and adherences were evaluated. The vaginal cavity was analyzed in terms of its healing, presence of infection, and dehiscence of the colpotomy. The position and effectiveness of the cystic duct clips were evaluated.

\section{Statistical analysis}

Data were collected on an evaluation card, tabulated, and compared among the groups. For the quantitative variables, Student's $t$-test and analysis of variance (ANOVA) were applied, using the post-hoc Tukey method for normally distributed variables. For categorical variables, Fisher's exact test was used. For all other variables, the Kruskal-Wallis test was used. In all analyses, the significance level was $\mathrm{p} \leq 0.05$.

\section{Results}

All of the procedures were successfully performed as proposed for each group. After the animals were returned to their bays, a team of veterinarians from CETEC tracked and observed 
them daily for 7 DPO, according to a control contained on each perioperative evaluation card as previously developed by the researchers. The animals were euthanized and necropsied on 7
DPO. We compared the animals' weights before the surgery and after euthanasia (Table 1). Only the laparoscopy group showed a marked weight gain in the postoperative period $(\mathrm{p}=0.046)$.

TABLE 1 - Initial and final weights of the animals in all groups.

\begin{tabular}{|c|c|c|c|c|c|c|}
\hline Groups & Variable & $\mathrm{n}$ & Average & $\mathrm{CI}$ & $95 \%$ & P-value \\
\hline \multirow[b]{2}{*}{ Laparoscopy } & Initial weight & 7 & 24.71 & 22.46 & 26.96 & \multirow[b]{2}{*}{0.046} \\
\hline & Final weight & 7 & 25.42 & 23.30 & 27.55 & \\
\hline \multirow{2}{*}{ Laparotomic Acess } & Initial weight & 7 & 26.14 & 23.34 & 28.93 & \multirow{2}{*}{0.842} \\
\hline & Final weight & 7 & 26.42 & 23.14 & 29.71 & \\
\hline \multirow{2}{*}{ Sham } & Initial weight & 5 & 26.4 & 22.82 & 29.97 & \multirow{2}{*}{0.846} \\
\hline & Final weight & 5 & 26.6 & 23.48 & 29.71 & \\
\hline \multirow{2}{*}{ NOTES } & Initial weight & 7 & 30.85 & 29.73 & 31.98 & \multirow{2}{*}{0.838} \\
\hline & Final weight & 7 & 30.71 & 28.96 & 32.46 & \\
\hline \multirow{2}{*}{ Single port } & Initial weight & 7 & 25.42 & 22.28 & 28.58 & \multirow{2}{*}{1.00} \\
\hline & Final weight & 7 & 24.85 & 22.41 & 28.44 & \\
\hline
\end{tabular}

Key: $\mathrm{n}$ - Sample size; CI - 95\% confidence interval. P-values were determined by paired Student's $t$-test.

Table 2 shows the results for perioperative clinical and hemodynamic parameters and postoperative times for the two groups. The anesthetic and operative times were higher in the vaginal NOTES group compared to the other groups ( $\mathrm{p}<0.001)$. The extubation time was statistically significant only for the laparoscopic group compared to the NOTES group. There were no statistical differences for the deambulation time, feeding time, $\mathrm{HR}$, or $\mathrm{O}_{2} \mathrm{SA}$ results among the groups. The $\mathrm{RR}$ was highest in the laparotomy group $(\mathrm{p}<0.01)$, and it differed significantly between the Laparotomy and the sham and vaginal NOTES groups.

TABLE 2 - Intraoperative parameters by study group.

\begin{tabular}{|c|c|c|c|c|c|c|}
\hline Parameter & Laparoscopy & Laparotomy & Sham & NOTES & Single port & P-value \\
\hline Anesthesia time (min.) & $84.85 \pm 21.03$ & $89.28 \pm 28.41$ & $74.00 \pm 10.46$ & $176.28 \pm 38.78$ & $105.4 \pm 18.18$ & $<0.001 *$ \\
\hline Extubation time (min.) & $14.85 \pm 9.29$ & $27.85 \pm 17.14$ & $66.85 \pm 42.44$ & $37.00 \pm 20.70$ & $19.28 \pm 3.14$ & $0.0377 * *$ \\
\hline Feeding time (min.) & $82.28 \pm 54.25$ & $94.00 \pm 30.29$ & $68.40 \pm 14.87$ & $102.23 \pm 62.94$ & $59.28 \pm 12.05$ & $0.3767^{*}$ \\
\hline Heart rate (min.) & $85.00 \pm 10.92$ & $89.85 \pm 20.07$ & $105.40 \pm 21.91$ & $87.00 \pm 27.51$ & $84.57 \pm 12.90$ & $0.3767 *$ \\
\hline Respiratory rate (rpm) & $14.71 \pm 7.04$ & $26.42 \pm 6.87$ & $13.40 \pm 3.84$ & $13.14 \pm 2.54$ & $16.00 \pm 1.41$ & $0.0007 * * *$ \\
\hline
\end{tabular}

Key: Data are reported as the mean \pm standard deviation. P-values were determined by *ANOVA, **Kruskal-Wallis, or ***Friedman. 
Table 3 shows the number of intraoperative complications and necropsy findings for each group. Only minor intraoperative complications were observed for all groups, such as perforation of the bile duct and bleeding in the liver bed. There was a lesion of the tail of the spleen in the vaginal NOTES group. Only adherences in the front abdominal wall and in the liver bed were found by necropsy. In the NOTES group, there were two cases of adherence in the back vaginal wall. All of the clips were positioned properly. There were no cases of fistula or intracavity abscess.

TABLE 3 - Intraoperative complications and necropsy findings for the study groups.

Group

Complication

Laparoscopy $\begin{gathered}\text { Laparotomic } \\ \text { Access }\end{gathered}$ NOTES Single portal P-value

$\begin{array}{cccccc}\text { Bleeding } & 1 & 2 & 1 & 0 & 0.883 \\ \text { Perforation } & 0 & 3 & 3 & 1 & 0.219\end{array}$

$\begin{array}{cccccc}\text { Adherence in the front abdominal wall } & 4 & 6 & 3 & 4 & 0.530 \\ \text { Adherence in the liver bed } & 4 & 7 & 7 & 2 & 0.079 \\ \text { Adherence in the back vaginal wall } & 0 & 0 & 3 & 0 & 0.079\end{array}$

Key: Data are reported as the number of events. P-values were determined by Fisher's Exact Test.

Tables 4, 5, and 6 show the results for the PCT, CRP, evaluation periods (preoperative, immediately postoperative, 2 and IFN- $\gamma$ values, respectively, in the different groups at different $\quad$ DPO, and 7 DPO).

TABLE 4 - Procalcitonin levels compared between study groups and between times.

\begin{tabular}{|c|c|c|c|c|c|c|}
\hline Time & Laparoscopy & Laparotomic Acess & Sham & NOTES & Single port & $\mathrm{P}$-value** \\
\hline Preoperative & 7.74 (1.67) & $8.11(0.57)$ & $8.65(0.67)$ & $8.22(0.58)$ & $8.1(1.67)$ & 0.1754 \\
\hline $2 \mathrm{DPO}$ & $6.43(2.13)$ & $8.11(0.92)$ & $8.3(0.24)$ & $8.02(0.35)$ & $9.22(1.82)$ & 0.1495 \\
\hline $\mathrm{P}$-value $* * *$ & 0.3598 & 0.4093 & 0.7819 & 0.1837 & 0.7633 & \\
\hline
\end{tabular}

Key: Data are reported as median (interquartile range). PO - Postoperative; DPO - Days postoperation. P-values in bottom row compare values in the same group between different time-points. P-values in the right-most column compare values at the same time point between groups. P-values were determined by ** Kruskal-Wallis or ***Friedman.

TABLE 5 - Comparison of C-reactive protein values between times and between groups.

\begin{tabular}{|c|c|c|c|c|c|c|}
\hline Time & Laparoscopy & Laparotomic acess & Sham & NOTES & Single port & $\mathrm{P}$-value ** \\
\hline Preoperative & 49.07 (93.17) & $56.02(81.79)$ & $210.84(187.99)$ & $191.66(132.78)$ & $109.72(245.81)$ & 0.5289 \\
\hline Immediate PO & $54.95(73.32)$ & $64.61(32.65)$ & $180.87(167.08)$ & $148.85(142.89)$ & $102.19(93.71)$ & 0.4211 \\
\hline $2 \mathrm{DPO}$ & $206.16(116.06)$ & $207.68(68.41)$ & $201.37(88.35)$ & $248.77(77.52)$ & $86.46(72.06)$ & 0.0044 \\
\hline $7 \mathrm{DPO}$ & $61.02(151.73)$ & $206.89(145.17)$ & 186.07 (61.62) & $132.41(180.53)$ & $95.15(172.6)$ & 0.2277 \\
\hline P-value $* * *$ & 0.0680 & 0.0010 & 0.0320 & 0.0210 & 0.7736 & \\
\hline
\end{tabular}

Key: Data are reported as median (interquartile range). IR - Interquartile range; PO - Postoperative; DPO - Days postoperation. Pvalues in bottom row compare values in the same group between different time-points. P-values in the right-most column compare values at the same time point between groups. P-values were determined by **Kruskal-Wallis or ***Friedman. 
Evaluation of systemic inflammatory responses in cholecystectomy by means of access. Single-port umbilical incision, transvaginal NOTES, laparoscopy and laparotomy

TABLE 6 - Comparison of interferon gamma (IFN- $\sigma$ ) levels between times and between study groups.

\begin{tabular}{ccccccc}
\hline Time & Laparoscopy & Laparotomy & Sham & NOTES & Single port & P-value** \\
\hline Preoperative & $4.2(3.39)$ & $3.45(9.6)$ & - & $5.72(4.3)$ & $3.1(2.8)$ & 0.8644 \\
Immediate PO & $5.37(2.93)$ & $6.55(9.68)$ & - & $5.92(5.66)$ & $8.5(55.4)$ & 0.6333 \\
2 DPO & $3.82(1.05)$ & $6.69(9.82)$ & - & $4.635(2.96)$ & $2(3.8)$ & 0.2937 \\
7 DPO & $5.69(2.42)$ & $7.92(5.47)$ & - & $2.92(4.94)$ & $3.4(9.2)$ & 0.4035 \\
P-value & 0.0710 & 0.0874 & - & 0.8766 & 0.2085
\end{tabular}

Key: Data are reported as median (interquartile range). PO - Postoperative; DPO - Days postoperation. P-values in bottom row compare values in the same group between different time-points. P-values in the right-most column compare values at the same time point between groups. P-values were determined by **Kruskal-Wallis or ***Friedman.

Table 7 compares the groups in terms of anesthesia, surgical, and extubation times. The NOTES group showed longer average anesthesia time $(\mathrm{p}<0.001)$ and surgical time $(\mathrm{p}<0.001)$ after surgery compared to the laparoscopy and Laparotomy groups. But, the results were not significant compared with single port incision group. $(\mathrm{p}<0.05)$. The greater surgical time needed for the NOTES group influenced post-anesthetic recovery, leading to a higher extubation time $(\mathrm{p}=0.0377)$ compared to the laparoscopic group. The postoperative deambulation and feeding times did not show significant differences among the groups.

TABLE 7 - Anesthesia, surgical, extubation, deambulation and feeding times by study group.

\begin{tabular}{ccccccc}
\hline Parameter & Laparoscopy & Laparotomy & Sham & NOTES & Single port & P-value \\
\hline Anesthesia time (min.) & $84.85 \pm 21.03$ & $89.28 \pm 28.41$ & $74.00 \pm 10.46$ & $176.28 \pm 38.78$ & $105.4 \pm 18.18$ & $<\mathbf{0 . 0 0 1 *}$ \\
Surgical time (min.) & $49.00 \pm 16.53$ & $46.28 \pm 17.04$ & - & $139.57 \pm 38.56$ & $56.28 \pm 18.64$ & $<\mathbf{0 . 0 0 1 *}$ \\
Extubation time (min.) & $14.85 \pm 9.29$ & $27.85 \pm 17.14$ & $66.85 \pm 42.44$ & $37.00 \pm 20.70$ & $19.28 \pm 3.14$ & $\mathbf{0 . 0 3 7 7 *}$ \\
Deambulation time (min.) & $83.57 \pm 54.22$ & $66.85 \pm 42.44$ & $65.08 \pm 15.51$ & $110.85 \pm 65.26$ & $57.28 \pm 15.70$ & $0.1560 * *$ \\
Feeding time (min.) & $82.28 \pm 54.25$ & $94.00 \pm 30.29$ & $68.40 \pm 14.87$ & $102.23 \pm 62.94$ & $59.28 \pm 12.05$ & $0.3767 *$ \\
\hline
\end{tabular}

Key: Data are reported as mean \pm standard deviation. P-values were determined by *ANOVA or **Kruskal-Wallis.

\section{Discussion}

Surgical knowledge and technological advances have contributed to improve surgery while reducing surgical trauma. The concept of MIS represents a fusion between the desires for less traumatic procedures and fewer incisions ${ }^{8}$. In the development of MIS techniques, surgeons have moved from open to laparoscopic surgery using multiple small incisions, with newer techniques including NOTES and single-port access approaches. These new techniques reduce surgical trauma, lessen pain in the immediate postoperative period, shorten the hospital stay duration, and allow a "scarless" aesthetic result ${ }^{8}$. However, technologies were not initially available for these new modes of access, and surgical teams were not experienced with the approaches. As a result, techniques using various degrees of laparoscopic assistance are only now becoming more common, as the procedures become safer and more feasible.

The single-port technique retains the potential benefits of the NOTES access method, as both approaches eliminate the risk of infection associated with deliberate visceral puncture. It also reserves the possibility of using material from conventional laparoscopic surgery ${ }^{8}$. Substantial efforts have been made towards designing new instruments, trocars, and optics to facilitate performing these procedures with a single trocar through the umbilical scar. As a result of this progress, increasing numbers of laparoscopic surgeons are interested in these new techniques. However, there is no scientific evidence of the real advantage or superiority of this new access approach ${ }^{19}$. On the other hand, 
authors have described the difficulty of finding the anatomical structures because of the position of the instruments when introduced through a single port. Thus, new instruments have been developed to maintain the safety of the method and to reduce the effect of the loss of triangulation between the forceps. Angled forceps and modified camera lenses have been developed to ease instrumental collision ${ }^{19}$.

Single-port cholecystectomy has several advantages. First, the technique is similar to traditional laparoscopic cholecystectomy and, in fact, can be converted into conventional laparoscopy if needed. Second, it reduces the number of skin incisions and morbidities associated with them (e.g., lesions on the epigastric veins and arteries, temporary pain at the incision, and muscle spasms). Third, it provides better aesthetics because it is based around a natural scar (i.e., the umbilical scar). Fourth, compared to NOTES, single-port umbilical cholecystectomy is easy and safe. Finally, it is possible to use rigid instruments, as in conventional laparoscopic cholecystectomy ${ }^{19}$. Its disadvantages include: less triangulation of the instruments than in conventional laparoscopic surgery; lack of lateral retraction during anatomical dissection in Calot's triangle; parallel positioning of the instruments in the left and right hands, resulting in an agglomeration of instruments on the same axis; frequent instrument collision, requiring great coordination between the surgeon and the assistant who manipulates the camera; and complicated dissection of surgical structures through the single port compared to conventional laparoscopy ${ }^{19}$.

Pfluke et al. ${ }^{9}$ described an increase in published studies using the single-port umbilical access since 2007, based on a systematic review of the literature. Of the publications, $73 \%$ were small case series with fewer than 20 patients; $6 \%$ reported having more than 100 patients; and only 12\% included results comparing the techniques. Only one prospective and random study with 40 patients compared conventional laparoscopic to single-port cholecystectomy. They concluded that there is still a lack of data about the real benefit of this technique, and that randomized comparative studies are needed to validate its clinical benefits and to determine whether new complications are associated with it. Patients continue to accept conventional laparoscopy as a safe method, and aesthetic reasons should not be the main motivation for introducing the single-port mode of access. Some degree of resistance is always encountered when a new technique is introduced, and randomized controlled studies are needed to determine the safety, efficacy, and cost effectiveness of any new $\operatorname{method}^{20}$.

\section{Motivations for the design and experimental model}

We designed this study to compare the clinical and immunological responses with single-port umbilical and transvaginal NOTES access approaches for cholecystectomy to laparoscopic (as the gold standard) and laparotomic approaches in an experimental model in pigs. We compared completely transvaginal NOTES cholecystectomy with single-port laparoscopy, four-port laparoscopy, and laparotomy, the latter of which is known to produce a relatively greater inflammatory response. A sham group was included to reduce the possibility of bias and to quantify the basal immune response caused by presurgical stress and anesthesia. Other authors with similar questions and designs have also used a sham group ${ }^{21}$.

We chose the pig experimental model of cholecystectomy because of the anatomic similarity between the liver and bile ducts of humans and pigs. Cholecystectomy is the most common laparoscopic surgical procedure in the Western world. Approximately $15 \%$ to $20 \%$ of adults worldwide have gallstones. Each year, more than 750.000 cholecystectomies are performed in the United States. More than 190.000 and more than 50.000 are performed each year in Germany and the United Kingdom, respectively. An unknown number of cholecystectomies are performed each year in Brazil, although $11.7 \%$ to $19.3 \%$ of Brazilians may be affected by gallstones. Various authors have compared the magnitude of the inflammatory response, based on systemic cytokine levels, in cholecystectomy ${ }^{22}$. Studies have also proven the technical validity and safety of the NOTES and multipleand single-port laparoscopic approaches in cholecystectomy ${ }^{23}$.

\section{Pneumoperitoneum and IAP}

For the single-port and conventional laparoscopic approaches, we used the same equipment and same laparoscopic insufflator control, with the pneumoperitoneum maintained between 10 and $14 \mathrm{mmHg}$. The only variation was that, in the single-port cases, $\mathrm{CO}_{2}$ was introduced into the same port that the operating equipment entered. In the NOTES group, the pneumoperitoneum was generated with ambient air by the endoscopic insufflator, also maintaining an IAP between 10 and $14 \mathrm{mmHg}$. However, because the equipment did not have a mechanism for rigorously controlling and monitoring IAP, the surgeon manually controlled the IAP. Aspiration of air was promoted when the IAP exceeded $14 \mathrm{mmHg}$, as measured through an exclusive endoscopic channel. Although various authors have used pneumoperitoneum with ambient air ${ }^{24}$, pneumoperitoneum with $\mathrm{CO}_{2}$ seems to play an important 
role in the improved immunologic response of laparoscopy over Laparotomy ${ }^{24} \cdot \mathrm{CO}_{2}$ is easily absorbed, and the reduced local $\mathrm{pH}$ creates an acidic environment, modulating the global immune response ${ }^{24}$.

A precise measurement of the pressure exerted by the pneumoperitoneum is essential to avoid the damaging effects of compartment abdominal syndrome ${ }^{24}$, particularly when the IAP exceeds $15 \mathrm{mmHg}^{24}$. Various experimental and clinical studies have described several systemic hemodynamic effects caused by high IAP, including tachycardia, increased blood pressure, peripheral vasoconstriction, cardiac deficit, and reduced circulating renal blood flow. Meireles et ll $^{25}$ obtained a good correlation for IAP measured through three methods of performing and maintaining pneumoperitoneum: the Veres needle, endoscope biopsy channel, and a 5-mm laparoscopic trocar in the abdominal wall. However, pneumoperitoneum generated by endoscopy can lead to large variations in IAP, increasing the risk of hemodynamic and respiratory compromise compared to a laparoscopic insufflator ${ }^{25}$.

\section{Surgical technique}

Technical aspects of the single-port approach. Singleport procedures can be related to specific technical difficulties, mainly related to retraction and triangulation, endoscopic materials, and hemostasis ${ }^{26}$. The ability to triangulate the forceps and to grip tissues for dissection are basic requirements in MIS. However, there is limited ability for instrument triangulation and tissue retraction in a single port, due to the position of the forceps and lens on the same axis. The surgeon must be able to visualize the anatomical structures from different perspectives and, preferably, away from the axis of the instrument. In response to these problems, flexible, articulated, and motorized instruments have been developed ${ }^{26}$.

Safe hemostasis has been a driver for technological research and is an integral component of the minimally invasive surgeon's arsenal. Safe and rapid hemostasis with the same efficiency as conventional laparoscopic surgery can be achieved in the single-port approach by using electrically instruments, clamps, and sutures. The use of instruments that permit the clips to be recharged reduces the need to change forceps and, therefore, the surgical time ${ }^{26}$. To make the single port, in this study, we used the SITRACC ${ }^{\circledR}$ device by Edlo. This commercially available disposable device is easy to use and install. The forceps had articulated ends and were developed especially for this access approach $^{27}$. Our initial experience with single-port umbilical surgery in the pilot group showed that using flexible and articulated forceps, as well as a silicone port, reduced the parallel positioning of the instruments, facilitating better instrument triangulation in the surgical field. A high-resolution $30^{\circ}$ lens also helped make the planned surgical method feasible.

Technical aspects of NOTES. All the NOTES procedures were performed in a completely transvaginal way. We used a flexible endoscope with the help of two extra-long forceps with arc grasps of $5 \mathrm{~mm}$, which were developed by our surgical team, based on the needs identified in the initial pilot studies. Under direct vision, the forceps and endoscope were moved into the abdominal cavity until they reached the upper right level. Using these forceps was necessary because surgery through natural orifices requires more resources than current endoscopes can offer ${ }^{28}$. Disposable endoscopes have only 2 narrow and parallel working channels that are very close together. These endoscopes are excessively flexible, which can make it difficult to grasp or handle structures or organs effectively ${ }^{28}$.

We performed the NOTES procedures successfully, with good spatial orientation, no conversions, and no complications. However, there were some difficulties. The vagina of the pig is tubular and narrow, which made it difficult to move the instruments laterally. This problem, in turn, meant that the instruments were not completely appropriate for this type of procedure. The development of articulated forceps (or, in this case, semicircular ones) facilitated the lateral movements of the distal end of the forceps, which was fundamental to forceps performance.

The artery and cystic duct were ligated with conventional clips used for endoscopic hemostasis. There were no problems with the quality of the ligature, and the autopsy revealed no complications related to clamping. Nevertheless, some authors have claimed that endoscopic clips are not safe for ligatures of the cystic duct and artery in humans. Hybrid techniques use a 5-mm trocar to pass through a laparoscopic clamper ${ }^{23}$. In contrast to our experiment, Gumbs et al..$^{29}$ had to modify the clips manually to increase the safety of the clamping.

The greatest difficulty faced by our team was dissection of the liver bed vessels. It may be that the surgical time was responsible for the greater operative time needed for the NOTES experiments compared to the other methods. The single-port approach took more time than conventional laparoscopy because of the greater difficulty in managing the instruments through a single port; dissection was limited to a single forceps, which was not the case when we used multiple ports. Because of the difficulty in achieving a perfect association of the movements of traction and contraction in the NOTES procedures, the electric current was greater than that needed for slight traction and contraction. As a 
result, there was a greater depth of dissection in the liver bed, and the small end branches of the hepatic veins were damaged, which prolonged and complicated the hemostasis process.

\section{Operative and anesthesia times}

Similar to our results, other authors have also found a higher operative time when comparing NOTES procedures with laparoscopy in experimental models ${ }^{30}$. In a systematic review, Pollard et al. ${ }^{8}$ compared the outcomes of NOTES and the single-port approach with the standard conventional fourport cholecystectomy. They examined 147 articles, with 36 about NOTES and 111 about the single-port approach. Among the 714 patients who underwent NOTES cholecystectomy, 3,989 patients who underwent single-port cholecystectomy, and 1,165 patients who underwent four-port cholecystectomy, the authors found that the operating time was similar for single-port and four-port cholecystectomy (79 and 83 minutes, respectively), but significantly higher (107 minutes) for NOTES cholecystectomy ${ }^{8}$. In our study, the anesthesia and surgical times with the single-port approach were intermediate between those of the laparoscopic, laparotomic, and NOTES approaches, and there was a significant difference in anesthesia time between the single-port and NOTES groups. This finding was also found in previous studies in humans, when comparing single-port and conventional laparoscopic surgeries ${ }^{31}$.

\section{Hemodynamic and respiratory evaluations}

We did not find a statistically significant difference for $\mathrm{HR}$ or $\mathrm{O}_{2} \mathrm{SA}$ among the laparoscopic, single-port, and vaginal NOTES groups. This outcome suggests that pneumoperitoneum with $\mathrm{CO}_{2}$ (laparoscopic) or ambient air (endoscopic) did not interfere with the animals' hemodynamic and ventilation states. However, the ambient air based pneumoperitoneum in the NOTES Group in contrast with the $\mathrm{CO} 2$ in the further groups could have influenced our results. We recognize that a more invasive and wider hemodynamic evaluation - involving such variables as the average blood pressure, pulmonary capillary pressure, and cardiac deficit — would have been more reliable. The RR was significantly higher in the laparotomy group compared to the sham and NOTES groups. This result may relate to the variation in the need for anesthetics and muscle blockers in each animal's anesthetic conduction, based on their study group.

\section{Peri- and post-operative complications}

All of the procedures were completely successfully. Although there were more perioperative perforation and bleeding in the liver bed of gallbladder) in the NOTES and laparotomy groups compared to the others, there was no significant difference between the groups. Gallbladder perforation is an expected outcome in cholecystectomy, even in open access surgery. In laparoscopy, perforation occurs in $18-36 \%$ of cases $^{32}$. Other experimental studies with a design similar to ours also did not have major complications $s^{21,30}$. We did not observe postoperative complications in the study groups, probably because of the small sample size and the reduced postoperative evaluation period used.

The higher incidence of lesions in the NOTES group may have been related to instrument handling by the surgical team, despite the level of experience of the endoscopist, and to the technical inadequacy of the endoscopic instruments. These lesions may have been caused by the parallel positioning of the instruments, which damaged the triangulation and surgical handling ${ }^{13}$. Endoscopes are currently being developed to address these limitations. These scopes, which are not yet commercially available, include the EndoSamurai (Olympus Corp., Tokyo, Japan) and the Anubiscope ${ }^{\circledR}$ (Karl Storz Corp., Tuttlingen, Germany).

\section{Clinical evolution}

All of the animals in all groups progressed satisfactorily, deambulated early, and ate normally. The comparison between the initial and final weights only showed a statistical difference for the laparoscopy group. These findings differ from those recorded by other studies, wherein all of the animals gained weight postoperatively ${ }^{30}$. One possible explanation for this discrepancy may be that the animals in this study were euthanized on 7 DPO. In previous studies, animals were euthanized much later (typically, 14 DPO) and, therefore, had more time to gain weight. However, in analyzing the animals' average weights, we observed that the initial weights in the laparoscopy group were, on average, lower than those in the other groups. This difference might have been due to an involuntary selection of younger animals in this group. As a result, they might have had a physiological rate of weight gain that was proportionally higher than that of the older animals.

To corroborate this hypothesis and to avoid the possibility that the laparoscopy group had less inflammation (less catabolism), we included a sham group. The initial average weight in the sham 
group was higher than in the laparoscopy group, and the sham animals showed no weight gain of statistical significance. The fact that animals from the NOTES group maintained the same weight as the others might suggest that this technique was not as catabolic as the others. In a prospective, randomized experimental study comparing distal pancreatectomy via transgastric NOTES versus laparoscopic access, Willigham et al..$^{33}$ did not observe any differences between the groups in terms of clinical parameters.

\section{Evaluation of inflammatory response}

Surgical trauma induces an acute inflammatory response that includes innate and adaptive immune factors. In response to an aggression, the damaged tissue releases antigenic products that activate cells involved the innate immune system, such as macrophages, neutrophils, natural killer lymphocytes, and endothelial cells. These activated cells synthesize different mediators, including cytokines ${ }^{33}$, direct the inflammatory response to the site of trauma or infection, and are essential to wound repair. However, excessive proinflammatory cytokine production from the trauma site can provoke hemodynamic instability or metabolic disorder.

Macrophages and monocytes at the trauma site initiate the production of proinflammatory cytokines, including TNF- $\alpha$ and IL-1 $\beta$, as part of the acute-phase response during and after surgery ${ }^{34}$. Other released cytokines include IL- $6^{35}$, which mediates the generation of acute-phase proteins, including CRP, fibrinogen, haptolglobin, antripsin-1, and PCT, among others ${ }^{36}$. IL-6 is the primary cytokine in the acute-phase response. Blood levels of IL-6 are sensitive and early markers of tissue trauma ${ }^{34}$. The plasma concentration of IL-6 has been shown to correlate with operative time, blood loss, and extent of tissue trauma. Prolonged and excessive increases in IL-6 have been associated with increases in morbidity, mortality, and even clinical deterioration in humans ${ }^{34}$.

Serum cytokine levels are commonly used as clinical markers to reflect the magnitude of surgical trauma and the seriousness of an infectious process. Previous clinical and experimental studies have evaluated trauma severity through the levels of acute-phase inflammatory mediators, revealing that laparoscopic surgery is significantly less invasive than the conventional Laparotomy method ${ }^{22}$. Clinical markers have also been used to study the immunological impact of the NOTES approach compared to the standard laparoscopic and single-port surgical methods ${ }^{15,21,30}$. However, in a previous study ${ }^{18}$, we found no statistically significant difference in IL-1 $\beta$, IL-6, and TNF- $\alpha$ levels between groups treated with transvaginal NOTES or a laparoscopic approach for cholecystectomy. Considering these findings, we resolved to use a different marker, to enable better diagnosis of the intensity of surgical trauma in our experimental model. Specifically, we compared the serum levels of IFN- $\gamma$, CRP, and PCT in pigs treated by cholecystectomy via the single-port umbilical, transvaginal NOTES, laparoscopic, or laparotomic approach.

As the most studied acute-phase protein in surgical trauma, CRP is a liver-synthesized protein that activates the complement cascade and stimulates phagocytosis by neutrophils and macrophages ${ }^{34}$. CRP offers a consistent and reliable global measurement of the acute-phase reaction after surgical trauma. CRP levels rise approximately four to 12 hours after surgery, peak at 24 to 72 hours, and return to baseline values at around two weeks, after control of the trauma ${ }^{37}$. PCT has also been used as an important acute-phase marker of inflammatory activity. The PCT level rises more quickly than the CRP level. PCT is also used for intensive therapies, abdominal surgeries, and followup in the evolution of transplants of organs such as the kidney and liver ${ }^{36}$. In response to infectious bacterial stimuli, serum PCT levels rise substantially. In the inflammatory response, PCT mediates chemotaxis functions, nitrous oxide synthesis, cytokine production, and so $\mathrm{on}^{38}$.

In this study, the CRP levels reached a maximum at 2 DPO for all animals in all groups, with the exception of the single-port group. In the latter group, the CRP levels at 2 DPO were lower than the preoperative levels. We found no statistically significant difference in the IFN- $\gamma$ levels among the laparoscopic, laparotomic, vaginal NOTES, and single-port groups, or among the different time points. The sham group was not included in the statistical analysis of the IFN- $\gamma$ because of technical problems during the course of biochemical tests. To the best of our knowledge, no previous study has evaluated the serum levels of IFN- $\gamma$ in the NOTES procedures. We did not find any significant differences in PCT levels among the five groups or at different times. Based on the acute-phase inflammatory response, we were unable to conclude whether the NOTES procedure was less invasive than laparoscopic or single-port techniques. Our results suggest that the NOTES approach is, at least, not more invasive. In this study we employed other inflammatory markers used in clinical practice for the purpose of judging whether some of them could signal a smaller response in relation to minimally invasive procedures, mostly using a marker as the pro calcitonin, which has been widely used for this purpose. But our results were not statistically significant for demonstrating this event. We believe that new studies in this regard should be made to clarify this point. 


\section{Conclusions}

The single-port umbilical and transvaginal NOTES access approaches were feasible and safe compared to laparoscopic and laparotomy for cholecystectomy. No significant inflammatory response between the analyzed groups based on the serum levels of C-reactive protein, procalcitonin, and interferon gamma was observed.

\section{References}

1 Buttenschoen K, Fathimani K, Buttenschoen DC. Effect of major abdominal surgery on the host immune response to infection. Curr Opin Infect Dis. 2010 Jun;23(3):259-67. doi: 10.1097/ QCO.0b013e32833939cb.

2 Corrigan M, Cahill RA, Redmond HP. The immunomodulatory effects of laparoscopic surgery. Surg Laparosc Endosc Percutan Tech. 2007 Aug;17(4):256-61. PMID: 17710044.

3 Yamamoto T, Umegae S, Kitagawa T, Matsumoto K. Intraperitoneal cytokine productions and their relationship to peritoneal sepsis and systemic inflammatory markers in patients with inflammatory bowel disease. Dis Colon Rectum. 2005 May;48(5):1005-15. PMID: 15785898.

4 Vittimberga FJ, Jr., Foley DP, Meyers WC, Callery MP. Laparoscopic surgery and the systemic immune response. Ann Surg. 1998 Mar;227(3):326-34. PMID: 9527054.

5 Hackam DJ, Rotstein OD. Host response to laparoscopic surgery: mechanisms and clinical correlates. Can J Surg. 1998 Apr;41(2):10311. PMID: 9575992.

6 Seven R, Barbaros U. Needloscopy-assisted transvaginal cholecystectomy. Surg Laparosc Endosc Percutan Tech. 2009 Apr;19(2):e61-3. doi: 10.1097/SLE.0b013e31819caa22.

7 Peterson CY, Ramamoorthy S, Andrews B, Horgan S, Talamini M, Chock A. Women's positive perception of transvaginal NOTES surgery. Surg Endosc. 2009 Aug;23(8):1770-4. doi: 10.1007/ s00464-008-0206-4.

8 Pollard JS, Fung AK, Ahmed I. Are natural orifice transluminal endoscopic surgery and single-incision surgery viable techniques for cholecystectomy? J Laparoendosc Adv Surg Tech A. 2012 JanFeb;22(1):1-14. doi: 10.1089/lap.2011.0341.

9 Pfluke JM, Parker M, Stauffer JA, Paetau AA, Bowers SP, Asbun HJ, Smith CD. Laparoscopic surgery performed through a single incision: a systematic review of the current literature. J Am Coll Surg. 2011 Jan;212(1):113-8. doi: 10.1016/j.jamcollsurg.2010.09.008.

10 Lehman AC, Dumpert J, Wood NA, Redden L, Visty AQ, Farritor S, Varnell B, Oleynikov D. Natural orifice cholecystectomy using a miniature robot. Surg Endosc. 2009 Feb;23(2):260-6. doi: 10.1007/ s00464-008-0195-3.

11 Rattner D, Kalloo A, ASGE/SAGES Working Group. ASGE/SAGES Working Group on Natural Orifice Translumenal Endoscopic Surgery. October 2005. Surg Endosc. 2006 Feb;20(2):329-33. PMID: 16402290.

12 Cuadrado-Garcia A, Noguera JF, Olea-Martinez JM, Morales R, Dolz C, Lozano L, Vicens JC, Pujol JJ. Hybrid natural orifice transluminal endoscopic cholecystectomy: prospective human series. Surg Endosc. 2011 Jan;25(1):19-22. doi: 10.1007/s00464-010-1121-z.

13 Niu J, Song W, Yan M, Fan W, Niu W, Liu E, Peng C, Lin P, Li P, Khan AQ. Transvaginal laparoscopically assisted endoscopic cholecystectomy: preliminary clinical results for a series of 43 cases in China. Surg Endosc. 2011 Apr;25(4):1281-6. doi: 10.1007/ s00464-010-1360-Z
14 Zornig C, Mofid H, Emmermann A, Alm M, von Waldenfels HA, Felixmuller C. Scarless cholecystectomy with combined transvaginal and transumbilical approach in a series of 20 patients. Surg Endosc. 2008 Jun;22(6):1427-9. doi: 10.1007/s00464-008-9891-2.

15 McGregor CG, Sodergren MH, Aslanyan A, Wright VJ, Purkayastha S, Darzi A, Paraskeva P. Evaluating systemic stress response in single port vs. multi-port laparoscopic cholecystectomy. J Gastrointest Surg. 2011 Apr;15(4):614-22. doi: 10.1007/s11605-011-1432-y.

16 Ponsky TA. Single port laparoscopic cholecystectomy in adults and children: tools and techniques. J Am Coll Surg. 2009 Nov;209(5):e1-6. doi: 10.1016/j.jamcollsurg.2009.07.025.

17 Duron VP, Nicastri GR, Gill PS. Novel technique for a single-incision laparoscopic surgery (SILS) approach to cholecystectomy: singleinstitution case series. Surg Endosc. 2011 May;25(5):1666-71. doi: 10.1007/s00464-010-1374-6.

18 Vieira JP, Linhares MM, Caetano EM, Jr., Moura RM, Asseituno V, Fuzyi R, Girao MJ, Ruano JM, Goldenberg A, de Jesus LFG, Matos D. Evaluation of the clinical and inflammatory responses in exclusively NOTES transvaginal cholecystectomy versus laparoscopic routes: an experimental study in swine. Surg Endosc. 2012 Nov;26(11):3232-44. doi: 10.1007/s00464-012-2329-x.

19 Podolsky ER, Curcillo PG, 2nd. Reduced-port surgery: preservation of the critical view in single-port-access cholecystectomy. Surg Endosc. 2010 Dec;24(12):3038-43. doi: 10.1007/s00464-010-10813.

20 Monkhouse SJ, Court EL, Beard LA, Bunni J, Burgess P. A retrospective wound review of standard four-port laparoscopic cholecystectomy: is there need for single-port laparoscopic surgery? Surg Endosc. 2012 Jan;26(1):255-60. doi: 10.1007/s00464-011-1863-2.

21 Fan JK, Tong DK, Ho DW, Luk J, Law WL, Law S. Systemic inflammatory response after natural orifice translumenal surgery: transvaginal cholecystectomy in a porcine model. JSLS. 2009 JanMar;13(1):9-13. PMID: 19366533.

22 Grande M, Tucci GF, Adorisio O, Barini A, Rulli F, Neri A, Franchi F, Farinon AM. Systemic acute-phase response after laparoscopic and open cholecystectomy. Surg Endosc. 2002 Feb;16(2):313-6. PMID: 11967686.

23 Asakuma M, Perretta S, Allemann P, Cahill R, Con SA, Solano C, Pasupathy S, Mutter D, Dallemagne B, Marescaux J. Challenges and lessons learned from NOTES cholecystectomy initial experience: a stepwise approach from the laboratory to clinical application. J Hepatobiliary Pancreat Surg. 2009;16(3):249-54. doi: 10.1007/ s00534-009-0089-3.

24 Trunzo JA, McGee MF, Cavazzola LT, Schomisch S, Nikfarjam M, Bailey J, Mishra T, Poulose BK, Lee YJ, Ponsky JL, Marks JM. Peritoneal inflammatory response of natural orifice translumenal endoscopic surgery (NOTES) versus laparoscopy with carbon dioxide and air pneumoperitoneum. Surg Endosc. 2010 Jul;24(7):1727-36. doi: 10.1007/s00464-009-0839-y.

25 Meireles O, Kantsevoy SV, Kalloo AN, Jagannath SB, Giday SA, Magno P, Shih SP, Hanly EJ, Ko CW, Beitler DM, Marohn MR. Comparison of intraabdominal pressures using the gastroscope and laparoscope for transgastric surgery. Surg Endosc. 2007 Jun;21(6):998-1001. PMID: 17404796.

26 Gill IS, Advincula AP, Aron M, Caddedu J, Canes D, Curcillo PG, 2nd, Desai MM, Evanko JC, Falcone T, Fazio V, Gettman M, Gumbs AA, Haber GP, Kaouk JH, Kim F, King SA, Ponsky J, Remzi F, Rivas H, Rosemurgy A, Ross S, Schauer P, Sotelo R, Speranza J, Sweeney J, Teixeira J. Consensus statement of the consortium for laparoendoscopic single-site surgery. Surg Endosc. 2010 Apr;24(4):762-8. doi: 10.1007/s00464-009-0688-8.

27 Skinovsky J, Martins MV, Chibata M, Cavalieri R, Tsumanuma F, Falcão D. Comparison between single trocar access (SITRACC) 
cholecysctomy and conventional laparoscopic cholecysctomy: one year follow-up. Bras J Video-Sur. 2012 Jan-Mar;5(1):3-8.

28 Horgan S, Cullen JP, Talamini MA, Mintz Y, Ferreres A, Jacobsen GR, Sandler B, Bosia J, Savides T, Easter DW, Savu MK, Ramamoorthy SL, Whitcomb E, Agarwal S, Lukacz E, Dominguez G, Ferraina P. Natural orifice surgery: initial clinical experience. Surg Endosc. 2009 Jul;23(7):1512-8. doi: 10.1007/s00464-009-0428-0.

29 Gumbs AA, Fowler D, Milone L, Evanko JC, Ude AO, Stevens P, Bessler M. Transvaginal natural orifice translumenal endoscopic surgery cholecystectomy: early evolution of the technique. Ann Surg. 2009 Jun;249(6):908-12. doi: 10.1097/SLA.0b013e3181a802e2.

30 Suzuki K, Yasuda K, Kawaguchi K, Yoshizumi F, Inomata M, Shiraishi N, Kitano S. Cardiopulmonary and immunologic effects of transvaginal natural-orifice transluminal endoscopic surgery cholecystectomy compared with laparoscopic cholecystectomy in a porcine survival model. Gastrointest Endosc. 2010 Dec;72(6):12418. doi: 10.1016/j.gie.2010.08.038.

31 Raakow R, Jacob DA. Single-Incision Cholecystectomy in about 200 Patients. Minim Invasive Surg. 2011;2011:915735. doi: 10.1155/2011/915735.

32 Mohiuddin K, Nizami S, Fitzgibbons RJ, Jr., Watson P, Memon B, Memon MA. Predicting iatrogenic gall bladder perforation during laparoscopic cholecystectomy: a multivariate logistic regression analysis of risk factors. ANZ J Surg. 2006 Mar;76(3):130-2. PMID: 16626349

33 Willingham FF, Gee DW, Sylla P, Kambadakone A, Singh AH, Sahani D, Mino-Kenudson M, Rattner DW, Brugge WR. Natural orifice versus conventional laparoscopic distal pancreatectomy in a porcine model: a randomized, controlled trial. Gastrointest Endosc. 2009 Oct;70(4):740-7. doi: 10.1016/j.gie.2009.03.021.

34 Schwenk W, Jacobi C, Mansmann U, Bohm B, Muller JM. Inflammatory response after laparoscopic and conventional colorectal resections results of a prospective randomized trial. Langenbecks Arch Surg. 2000 Jan;385(1):2-9. PMID: 10664112.

35 Baigrie RJ, Lamont PM, Kwiatkowski D, Dallman MJ, Morris PJ. Systemic cytokine response after major surgery. Br J Surg. 1992 Aug;79(8):757-60. PMID: 1393463.

36 van Ree RM, de Vries AP, Oterdoom LH, Seelen MA, Gansevoort RT, Schouten JP, Struck J, Navis G, Gans RO, van der Heide JJ, van Son WJ, Bakker SJ. Plasma procalcitonin is an independent predictor of graft failure late after renal transplantation. Transplantation. 2009 Jul 27;88(2):279-87. doi: 10.1097/TP.0b013e3181ac9ea0.

37 Halevy A, Lin G, Gold-Deutsch R, Lavi R, Negri M, Evans S,
Cotariu D, Sackier JM. Comparison of serum C-reactive protein concentrations for laparoscopic versus open cholecystectomy. Surg Endosc. 1995 Mar;9(3):280-2. PMID: 7597598.

38 Sexton PM, Christopoulos G, Christopoulos A, Nylen ES, Snider RH, Jr., Becker KL. Procalcitonin has bioactivity at calcitonin receptor family complexes: potential mediator implications in sepsis. Crit Care Med. 2008 May;36(5):1637-40. doi: 10.1097/ CCM.0b013e318170a554.

\section{Acknowledgements}

To Experimentation and Training Center for Experimental Surgery, Albert Einstein Israelite Hospital; veterinarian Valéria Chiba for the technical support toward this research; Salomão \& Zopi Diagnostic Medicine Laboratory, Drs. Gianfranco Zampieri and Patrícia Udiloff for their assistance with cytokine dosage.

\section{Correspondence:}

Elesiário Marques Caetano Júnior

Rua Tomas Carvalhal, 555/142

04006-001 São Paulo - SP Brasil

Tel.: (55 11)2440-9074

elesiario@terra.com.br

Received: Jun 12, 2015

Review: Aug 14, 2015

Accepted: Sep 14, 2015

Conflict of interest: none

Financial sources: CNPq and FAPESP

${ }^{1}$ Research performed at Division of Surgical Gastroenterology, Department of Surgery, Universidade Federal de São Paulo (UNIFESP), Brazil. Part of Master degree thesis, Postgraduate Program in Interdisciplinary Surgical Sciences, UNIFESP. Tutor: Gaspar de Jesus Lopes-Filho. 